\title{
Which antibiotic for hospital acquired pneumonia caused by MRSA?
}

In this Editorial (BMJ 2014;348:g1469, doi:10.1136/bmj.g1469), author John Muscedere cited a systematic review and

Cite this as: BMJ 2014;348:g2362

meta-analysis by AC Kalil and colleagues (reference 13). While

๑ BMJ Publishing Group Ltd 2014

Kalil's name was correct in the references section, in the text

of the article it was mistakenly spelt as "Khalil" in three places. 